\title{
Using Community-Based Participatory Research to Characterize Health and Lifestyle Behaviors of Montagnards, a Refugee-Origin Asian-American Subgroup
}

\author{
Sharon D. Morrison ${ }^{1}$ (D) S. Sudha ${ }^{2} \cdot$ Kunga Denzongpa $^{1} \cdot \mathrm{H}^{\prime}$ Yua Adrong $^{3} \cdot \mathrm{Kelsie} \mathrm{Bernot}^{4} \cdot$ Michele Malotky $^{5} \cdot$ \\ Maura Nsonwu ${ }^{6}$
}

Accepted: 9 September 2021 / Published online: 24 September 2021

(c) The Author(s), under exclusive licence to Springer Science+Business Media, LLC, part of Springer Nature 2021

\begin{abstract}
Montagnards, an indigenous multitribal refugee-origin population concentrated in North Carolina, remain an invisible, medically underserved, and socioeconomically underrepresented Asian American sub-group. Yet this group is resilient, with language diversity, rich cultural traditions and family caregiving in multigenerational households. Using community-based participatory research methods, we developed and administered a two-part survey to 144 Montagnard adults, documenting socioeconomic characteristics, health indicators and lifestyle behaviors. Forty-one percent of participants had no formal education, 76\% had little/no English proficiency and 28\% described having a very hard time paying their bills. Seventy-seven percent were overweight per BMI category, $79 \%$ had elevated blood pressure and $100 \%$ scored high for significant depressive symptoms. Participants reported high levels of physical inactivity and daily dietary intake of MSG. However, Montagnards reported limited tobacco and alcohol use, a diet of fresh vegetables and rice, and regular church attendance. These represent protective lifestyle behaviors and targets for culturally responsive health interventions.
\end{abstract}

Keywords Montagnards $\cdot$ Refugee-origin $\cdot$ Asian-American $\cdot$ Health $\cdot$ Lifestyle

\section{Introduction}

Sharon D. Morrison

sdmorri2@uncg.edu

1 Department of Public Health Education, University of North Carolina Greensboro, 437 Coleman Building, 1408 Walker Avenue, Greensboro, NC 27402-6170, USA

2 Department of Human Development and Family Studies, University of North Carolina Greensboro, 248 Stone Building, Greensboro, NC 27402-6170, USA

3 Montagnard Dega Association/Montagnard American Organization, Suite 10, 611 Summit Avenue, Greensboro, NC 27405, USA

4 Department of Biology, Barnes 211, North Carolina A \& T State University, 1601 E. Market Street, Greensboro, NC 27411, USA

5 Department of Biology, Guilford College, 5800 W. Friendly Avenue, Greensboro, NC 27410, USA

6 School of Social Work, North Carolina State University, 1911 Building 207, Campus Box 7639, Raleigh, NC 27695-8101, USA
The Montagnards (French for "mountain people") are unique, indigenous, multi-tribal people from the highlands of Vietnam. There are six main ethnic tribal groups-Jarai, Rhade, Bhnar, Koho, Bunong and Stieng-all existing as isolated mountain farmers and hunter-gatherers until the Vietnam War, when the US government recruited them as frontline soldiers for the US Army Special Forces [1]. Montagnards suffered retribution from the Vietnamese government, who destroyed their villages, stole their land and imprisoned family members [1]. Because of this history, they have deliberately chosen to be identified as Montagnard to distinguish them as an oppressed minority within Vietnam. Their strategic adoption of this colonial term is deeply rooted in the desire to (1) forge a common group identity and (2) communicate a sense of solidarity to outsiders irrespective of tribal affiliation or language spoken. The Department of State granted refugee status to Montagnards, with the first wave of individuals arriving in the U.S. in 1986 and subsequent waves during the 1990s and early 2000s [2]. 
In more recent years, Montagnards have come to the U.S. as immigrants through U.S. family reunification channels.

North Carolina now has the largest population of Montagnards (an estimated 12,000+) outside of Vietnam, with the vast majority residing in the Piedmont Triad region [3]. Despite $30+$ years of residence in the state, Montagnards remain poorly integrated due to residual trauma from war, language and culture differences, low socioeconomic status and low health literacy [4, 5]. Anecdotal accounts from families and case managers coupled with current research studies, indicate pervasive food insecurity, unfamiliarity and misunderstandings of Western medical systems and ongoing chronic health challenges that remain unaddressed by state and county level health and social service providers [6-8]. Further, the lack of representation in prominent demographic and health related databases creates a gap in data specific to this Asian immigrant/refugee population. Therefore, our study serves as one of a few to focus on Montagnards as a subgroup of the Asian American minority population.

The purpose of this paper is to present a snapshot of health and lifestyle factors of Montagnard refugees and immigrants residing in central North Carolina. The paper provides a sociodemographic profile and describes patterns in significant health indicators including blood pressure (BP) and body mass index (BMI), self-reported health status, chronic diseases and lifestyle behaviors (physical activity, smoking, alcohol use and diet) among this population. Asian Americans are often represented as a model minority whose economic and health indicators score above that of non-Hispanic Whites. However, this large ethnic category has great diversity across subgroups and is described as the most economically divided ethnic group in the nation, with some subgroups experiencing enormous disadvantages [9, 10]. Thus, this study adds to the minority health literature by providing information that characterizes chronic disease along with lifestyle risk factors among this medically underserved, lower socioeconomic status, immigrant/refugee origin Asian-American subgroup.

\section{Methods}

\section{Community-Based Participatory Research (CBPR) approach}

The data and analysis presented in this paper are part of a larger mixed-methods project, the Montagnard Hypertension Project, which was guided by CBPR principles. CBPR is an action-oriented process whereby (1) community-expressed priorities are central to the research, (2) community members function as co-researchers and co-learners and (3) the focus is on partnership and team building for mutual trust, respect, shared decision-making, reciprocity and "give backs" for community capacity building and empowerment [11]. CBPR is an ideal approach to conducting research with tribal communities because it incorporates their indigenous knowledge and ways of operating, highlights tribal diversity and the importance of understanding variations in values, ideas and practices within ethnic communities, and recognizes key gatekeepers who are central to establishing trusting relationships [12].

We used a CBPR approach when working with the Montagnard community because of their continued struggle with social integration and a dearth of published, culturally viable and pragmatic strategies for research and outreach with multi-tribal, multi-language communities. Following CBPR principles, community leaders prioritized the research focus to describing chronic disease presence in this community within the context of their historical experience with war, resettlement and integration, social invisibility, intergenerational conflicts and unfamiliarity with formal higher education processes. CBPR fostered a reciprocal relationship and supported a balance of power as researchers worked in concert with Montagnard community stakeholders [13]. For example, the project supported co-learning and capacity building, whereby researchers trained Montagnard youth and former Montagnard medical professionals (MMPs) in research ethics, human subject protection protocols and data collection procedures. In turn, community leaders, pastors and church elders educated researchers (i.e. the outsiders) about tribal dynamics, present-day religious life and community relations, including preferences for handling private community matters and tabooed subjects.

Through CBPR, researchers became more attentive to the power dynamics across generations, the diversity of attitudes towards health and wellbeing and gained greater understanding and respect for indigenous knowledge systems associated with illness and disease outcomes. Researchers were able to meet and facilitate conversations between youth and their elders, provide suggestions on strategies that could build consensus around action and promote the value and importance of youth involvement in health research and prevention outreach. Finally, CBPR's emphasis on reciprocity allowed for simultaneous data collection with tailored outreach, e.g. free flu vaccines, health screenings and community health fairs that connected primary care clinicians with some of the sickest members of the community $[12,14]$.

\section{Survey and Research Participants}

We developed a survey to explore chronic disease and health in partnership with the former MMPs, first generation college youth and community advocates. The survey included a combination of standard questionnaire measures used for Asian populations and questions derived from prior informal conversations and formative focus group discussions 
with Montagnard men and women on indigenous health practices [15]. The questions were translated by bilingual/ bicultural Montagnard college students into the three main Montagnard tribal languages (Rhade, Jarai and Bunong) and presented to a sample of community members for culturally sensitive refinement and face validity testing. The entire survey development process is outlined in detail in a previous publication [15].

The University's IRB and key Montagnard community stakeholders both approved the final survey and study protocols (Study\# 14-0242) and data collection occurred in 2015-2016. Participants included 144 Montagnard men and women aged 18 years and older living in Greensboro NC, the city with the largest concentration of Montagnards. Participants were recruited by convenience sampling, through word-of-mouth outreach by Montagnard churches, community organizations and bilingual/bicultural Montagnard college students. Informed consent procedures with participants included oral administration using simultaneous interpretation and videos in each of three major tribal languages (Rhade, Jarai and Bunong). Research teams, which included Montagnard college students trained in anthropometric and biological sample data collection protocols, administered the surveys in participants' preferred locations including Montagnard family homes and group meeting areas of Montagnard Christian churches. All respondents received two $\$ 10$ grocery store gift cards in appreciation for their willingness to participate.

The survey was completed in two sessions due to its length and complexity. First, 144 participants completed the biological section, which included BP, height and weight measurements and collection of saliva and scalp hair samples (to measure cortisol in saliva and scalp hair). We collected these biological data to ascertain information relevant to chronic diseases and biomarkers for stress experienced by the community. Analyzing stress biomarker data is outside the scope of the present paper and will be addressed in subsequent studies. Our research team followed up with participants within a few weeks, and 128 of the original 144 completed the behavioral section of the survey that comprised demographic, socioeconomic, health-related and lifestyle questions.

\section{Data Collection}

Health related measures reported in the present paper included BP, calculated by using systolic and diastolic measurements. BP measurements were collected twice, using an OMRON Intellisense BP Monitor model HEM907XL calibrated per manufacturer instructions. We fitted participants with an appropriately sized cuff, had them sit with feet uncrossed and flat on the ground, then took two BP readings each 3 min apart. The mean of these BP readings was summed and averaged to obtain a single measure, which was then dichotomized into systolic $<130$ and diastolic $<80$ as normal and measures $\geq 130 / 80$ as elevated BP [16]. Participants were also asked whether a doctor had diagnosed them with hypertension (Yes/No response) and if yes, were they taking "American" medication for the condition. Chronic disease prevalence included self-reported 'yes' or 'no' responses for chronic illnesses listed on the questionnaire. These illnesses were selected based on chronic diseases listed in the CDC's report on the leading causes of death in the U.S. in 2013 [17].

Formative research revealed that Montagnard adults were more inclined to talk about depressive symptoms, but less willing to engage in open conversations about post-traumatic stress disorder or mental health in general with "American outsiders". Our survey focused on depressive symptoms because (i) this was expressed as a community priority, (ii) we were conducting a large survey as a pilot effort and had to select only some domains, and (iii) there is lack of mental health services with capacity to respond to diverse English language learner communities in the area and thus limiting capacity to investigate additional mental health factors or offer support to participants reporting these challenges. Participants were therefore administered the CESD-8 depression scale to indicate depressive symptoms [18]. Perceived health status was measured by a single question asking participants to rate their overall health status on a 5-point scale $(1=$ excellent, $2=$ very good, $3=$ good, $4=$ fair, $5=$ poor), with higher rating indicative of poorer health status. Three height and weight measures were taken during the survey with a portable stadiometer and electronic scales. The mean of three readings was used to calculate BMI, contrasting those with $\mathrm{BMI} \geq 23$ as overweight (coded 1 ) vs those less than that (coded 0), as indicated for Asian populations [19, 20]. Preventative health care access was measured by one question where participants were asked to rate (mostly, sometimes, rarely) on the following question: 'I have a doctor I see regularly'.

Demographic and socioeconomic measures included gender (male/female) and age, tribal group within the Montagnard community, religious affiliation and factors indicating acculturation and socio-economic status: years in the U.S., education, employment status and English proficiency. Financial security was measured by a single question asking participants how hard it is to pay their bills with response options: 'very hard', 'quite hard', 'a little hard' and 'not hard at all'. Lifestyle behaviors included physical activity, measured using questions validated in a survey conducted among Vietnamese populations [21] and smoking and drinking habits assessed by a three-level categorical variable (daily, sometimes, never) [22]. Dietary practices were measured by asking the frequency of rice, noodle, vegetable, fast food, 
soda, sugar and MSG intake, where participants could select 'daily', 'sometimes' and 'never'.

Descriptive statistics (mean $\pm \mathrm{SD}$, frequencies, percentages) were analyzed to provide a profile of the sample from the listed categories using SPSS version 25 for analysis (IBM Corp., Armonk, NY).

\section{Results}

We report biological data from the 144 adults who first participated and behavioral information from the 128 who completed the second session.

\section{Demographic and Socioeconomic Characteristics}

The sample was slightly more female than male (about 53\% to $47 \%$ ), aged on average about 48 years, with most in the middle age group (45-64 years, Table 1). The majority were married or in a long-term partnership (85\%). Most (96\%) were born in Vietnam and affiliated with two main tribes: Rhade (52\%) and Jarai (34\%). About $45 \%$ had lived in the U.S. for 20 or more years. All reported (Protestant) Christianity as their religion and most attended church at least weekly. Just over $40 \%$ had no formal education and only $14 \%$ had high school or more education. Three-quarters (76\%) had little to no English language fluency. Sixty one percent were employed, with most (about $82 \%$ ) of those in full time employment. Despite this relatively high employment status, over half of respondents (53.6\%) reported a 'very' or 'quite' hard time paying their bills.

\section{Health Status}

Respondents' most often reported that their health was "Fair" (about 34\%), followed by "Good" (20\%). Eighteen percent (18\%) of participants perceived their health status as "Poor", with $15 \%$ reporting it as "Very Good. The lowest $(15 \%)$ reported status was "Excellent" (Table 2). This indicates a profile of lower self-rated health. However, almost $60 \%$ saw their regular doctor only sometimes or rarely. Respondents had a range of self-reported chronic conditions including depression (19\%), high cholesterol (18\%), heart problems (11\%) and diabetes (10\%). However, the CESD-8 depression scale measured in the survey indicated that $100 \%$ of the participants scored over 7.0, a cut off indicating significant distress [18]. About $76 \%$ of participants had a $\mathrm{BMI} \geq 23$, considered overweight for Asian populations. Fifty six percent of the participants (79 people) had elevated BP per the survey. Thirty-two percent of the total sample reported being diagnosed with hypertension and about $65 \%$ of those so diagnosed reported taking medication. In results not shown in a table, of the 79 people who had elevated BP in the survey, only 16 were taking medication. Further, of the 22 people who reported taking medication, 16 of them still had elevated BP during the survey, suggesting poor BP control.

\section{Lifestyle Behaviors}

The majority of respondents indicated they consumed neither tobacco nor alcohol (Table 3). Seventeen percent of participants reported no light to moderate physical activity and $65 \%$ reported no vigorous physical activity. Regarding dietary behaviors, the majority $(97 \%)$ of respondents consumed rice daily. 'American noodles' (e.g. packaged Ramen noodles) were consumed daily by about $58 \%$ of the participants while 'Asian noodles' (e.g. clear or rice noodles) were consumed much less frequently with only $4 \%$ reporting them as part of their daily diet. Daily consumption of vegetables was high $(85 \%)$, as was the daily consumption of MSG (78\%) used primarily as a seasoning. Participants reported occasional consumption of fast foods and soda at $74 \%$ and $56 \%$ respectively. Finally, $47 \%$ of respondents reported occasionally (sometimes) eating out at 'American' (e.g. McDonalds or Burger King) and 78\% at 'Asian' (e.g. Vietnamese Pho) restaurants.

\section{Discussion}

This study adds to the literature on the refugee/immigrant minority populations in the United States, by being the first to apply the CBPR approach to creating a snapshot of the health and lifestyle behaviors of the Montagnard community living in North Carolina. Published literature on this population is sparse, despite their arrival into the United States over 30 years ago. Thus, our study is unique and important in several ways as it (1) situates the Montagnard population within the CBPR literature, having been the first study to engage this Asian American subgroup and its diverse community stakeholders as co-researchers and co-learners, (2) documents important psychosocial and behavioral determinants of health and disease occurrence within this underserved population and (3) highlights lifestyle behaviors that play an important role in chronic disease prevention.

Our results strengthen the argument to disaggregate Asian American subgroups rather than considering all as lowhealth-risk 'model minorities'. The participants described in this study represent households with lower education, limited English proficiency and low socioeconomic status. To put these findings into perspective, in 2019, only $13 \%$ of Americans overall and 13\% of Asian Americans in the aggregate had not completed high school [23]. However, disaggregating the Asian group shows that a disproportionate number of Southeast Asian Americans had less than a 
Table 1 Demographic and socioeconomic characteristics of the sample

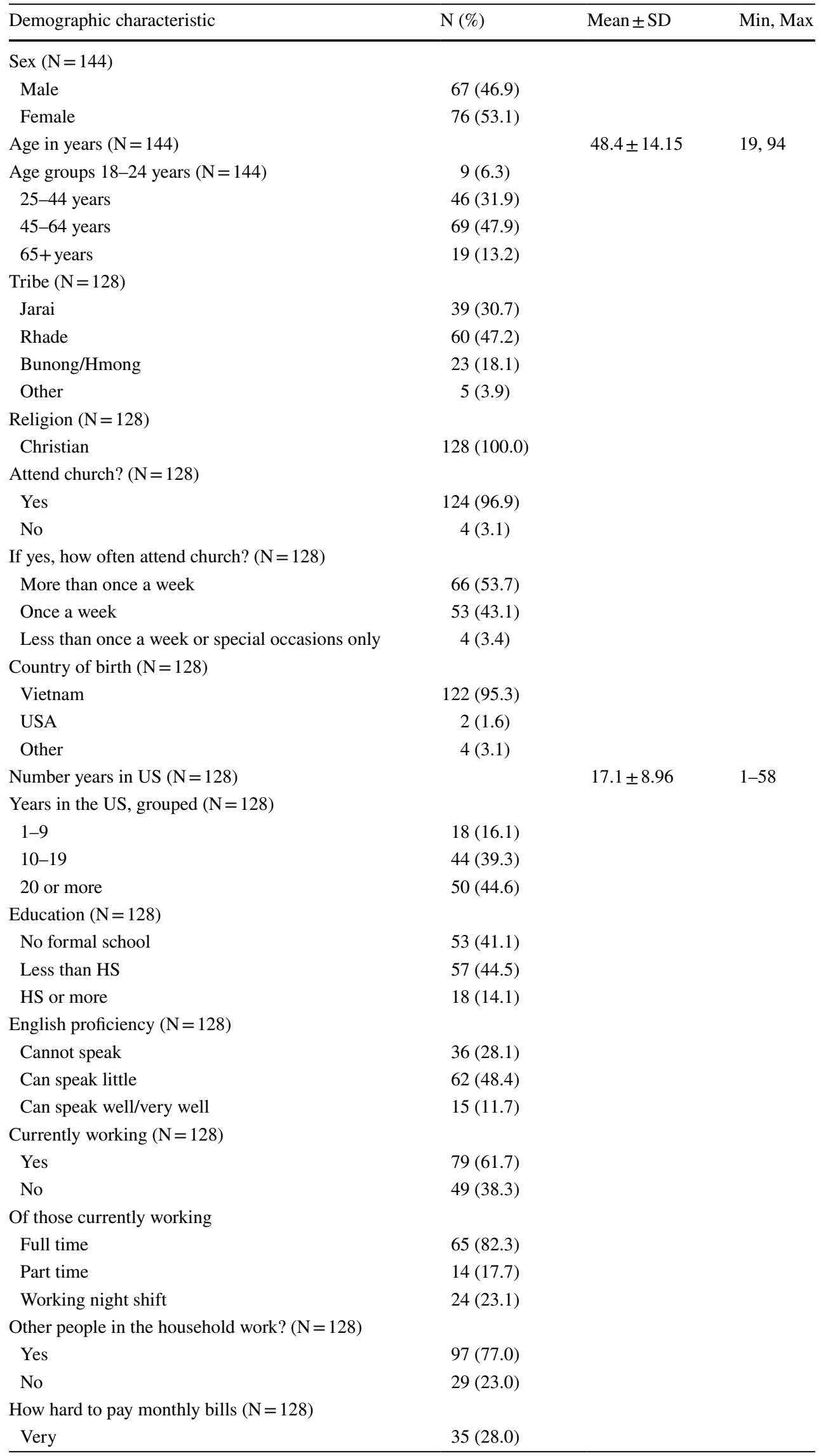


Table 1 (continued)

\begin{tabular}{|c|c|c|c|}
\hline Demographic characteristic & $\mathrm{N}(\%)$ & Mean \pm SD & Min, Max \\
\hline Quite & $32(25.6)$ & & \\
\hline Little & $33(26.4)$ & & \\
\hline Not at all & $25(20.0)$ & & \\
\hline \multicolumn{4}{|l|}{ Marital status $(\mathrm{N}=128)$} \\
\hline Married or partnered & $108(85.0)$ & & \\
\hline Not married or partnered & $19(15.0)$ & & \\
\hline
\end{tabular}

$\mathrm{N}=144$ if participants completed both halves of the survey; $\mathrm{N}=128$ if they completed only the first half

Table 2 Health and chronic disease profile of the sample

\begin{tabular}{|c|c|c|}
\hline Characteristic & $\mathrm{N}(\%)$ & Mean $\pm S D$, range \\
\hline \multicolumn{3}{|l|}{ Self-reported perceived health status $(\mathrm{N}=128)$} \\
\hline Excellent & $15(11.7)$ & \\
\hline Very good & $20(15.6)$ & \\
\hline Good & $26(20.3)$ & \\
\hline Fair & $44(34.4)$ & \\
\hline Poor & $23(18.0)$ & \\
\hline \multicolumn{3}{|l|}{ Self-reported health conditions $(\mathrm{N}=128)$} \\
\hline Heart problem & $15(12.4)$ & \\
\hline Stroke & $2(1.7)$ & \\
\hline Cancer & $4(3.3)$ & \\
\hline Diabetes & $13(11.0)$ & \\
\hline Kidney problem & $6(5.1)$ & \\
\hline High cholesterol & $21(17.6)$ & \\
\hline Depression & $22(18.6)$ & \\
\hline \multicolumn{3}{|l|}{ BMI } \\
\hline Normal & $32(22.5)$ & \\
\hline Overweight (BMI $\geq 23$ for Asians) & $110(77.5)$ & \\
\hline $\begin{array}{l}\text { High blood pressure }(\mathrm{N}=144) \text { (per } 2017 \text { ACC guidelines: sys- } \\
\text { tolic } \geq 130 \text { OR diastolic } \geq 80)\end{array}$ & $79(56.0)$ & \\
\hline "Normal” BP & $62(44.0)$ & \\
\hline \multicolumn{3}{|l|}{ Doctor diagnosed hypertension $(\mathrm{N}=128)$} \\
\hline Yes & $39(30.7)$ & \\
\hline No & $88(69.3)$ & \\
\hline \multicolumn{3}{|l|}{ If yes, does participant take "American medicine" $(\mathrm{N}=116)$} \\
\hline Yes & $22(65.6)$ & \\
\hline No & $12(35.3)$ & \\
\hline CESD-8 depression score $(\mathrm{N}=128)$ & & $18.65 \pm 3.40,11-24$ \\
\hline \multicolumn{3}{|l|}{ Sees a regular doctor $(N=128)$} \\
\hline Most of the time & $50(40.3)$ & \\
\hline Sometimes or rarely & $74(59.7)$ & \\
\hline
\end{tabular}

$\mathrm{N}=144$ if participants completed both halves of the survey; $\mathrm{N}=128$ if they completed only the first half high school education: Hmong 30.2\%, Laotian 30.2\%, and Cambodian $33.9 \%$ [24]. Similarly, in $2019,10.5 \%$ of all Americans were in poverty [25], but the percent of Asian Americans in poverty was $12.6 \%$, while poverty among Vietnamese (15\%), Lao (16.6\%), Hmong (26.1\%) and Cambodians (19.8\%) was much higher [24]. These factors remain challenges for working age and older adults in the
Montagnard community. Prior to relocation, many came from subsistence farming backgrounds, which required manual labor and promoted simple but healthier diets. However, the transition to a more urban lifestyle continues to be a difficult one as Montagnards are faced with exposure to unsafe and unhealthy physical environments including poor-quality housing [26]. Discussions with community informants 
Table 3 Lifestyle behaviors reported by the sample

$\mathrm{N}(\%)$

Current tobacco use in any form $(\mathrm{N}=128)$

Daily
Occasionally
Do not use
I don't know
Current alcohol consumption in any form $(\mathrm{N}=128)$
Daily

Daily

$6(4.8)$

$2(1.6)$

$115(92)$

2 (1.6)

Occasionally

$1(0.8)$

Do not take

I don't know

$15(11.8)$

$110(86.6)$

$1(0.8)$

Physical activity $(\mathrm{N}=128)$

Light/moderate

Often

Sometimes

43 (35.5)

Never

$57(47.1)$

21 (17.4)

Vigorous

Often

Sometimes

$26(21.1)$

17 (13.8)

Never

Dietary practices $(\mathrm{N}=128)$

Rice consumption

Daily

Sometimes

123 (96.9)

4 (3.1)

0

American noodle consumption

Daily

Sometimes

Never

Asian noodle consumption

Daily

Sometimes

Never

Vegetable consumption

Daily

Sometimes

Never

Fruit consumption

Daily

Sometimes

Never

Sugar consumption

Daily

Sometimes

Never

74 (58.7)

$52(41.3)$

0

$4(3.1)$

106 (83.5)

0

108 (85)

19 (15)

0

$28(22.2)$

94 (74.6)

$4(3.4)$

$12(10.0)$

$37(30.8)$

$71(59.2)$

$94(78.3)$

$20(15.7)$
Table 3 (continued)

\begin{tabular}{ll}
\hline & $\mathrm{N}(\%)$ \\
\hline Never & $6(5.0)$ \\
Eating out Asian restaurants & 0 \\
Daily & $100(78.7)$ \\
Sometimes & $27(21.3)$ \\
Never & \\
Eating out American restaurants & 0 \\
Daily & $60(47.2)$ \\
Sometimes & $67(52.8)$ \\
Never & \\
Fast food consumption & $2(1.6)$ \\
Daily & $94(74.0)$ \\
Sometimes & $31(24.4)$ \\
Never & \\
Soda consumption & $3(2.4)$ \\
Daily & $70(56.0)$ \\
Sometimes & $52(41.6)$ \\
Never &
\end{tabular}

$\mathrm{N}=144$ who completed the full survey; $\mathrm{N}=128$ if they did not complete the second part of the survey

indicate that many continue to be unaware of the important links between health status and lifestyle behaviors.

The findings in this study provide information that can be useful to both Montagnard community leaders and to U.S. health practitioners to identify education and outreach needs within this group. For example, our results show that a substantial proportion of Montagnards report experiencing chronic disease, including high BP and depression. Their self-reports were supplemented by survey data measures. These findings are consistent with those from a previous study conducted by Bertrand et al. [27]. It is unlikely that the elevated BP measured in the survey was due to a "white coat' effect, as the data were collected in familiar community settings by a multi-cultural, trained, student team. However, 39 participants reported that they had been diagnosed by a doctor with high BP but only 22 were taking medication for it and 16 of those still had elevated BP in the survey. This, coupled with the high percent with overweight BMI, indicates a Southeast Asian community at risk for cardiovascular disease [27]. In fact, in a large study from California examining cardiovascular disease risk, about $36 \%$ of Southeast Asians (not disaggregated further) ages $45-80$ years had been diagnosed with hypertension and about $18.7 \%$ had obesity [28]. Our results indicate substantial levels of unmet needs for primary health care and chronic disease risk reduction in the Montagnard community.

With all participants in the study reporting high levels of psychological distress, there appears to be a pressing 
need for culturally customized mental health services and family-centered interventions to alleviate pervasive social and economic stressors in this Asian American community. For comparison, during 2015-2016, serious psychological distress in the past 30 days was reported by $2.1 \%$ of Asian Americans aged 18 years and older overall [29]. However, few studies examine disaggregated Asian American groups, and report a wide variation in psychological outcomes. For example, severe psychological distress ranged from 2 to $14 \%$ among Vietnamese origin Americans in the studies reviewed by Yang and Mutchler [30]. Their study found an HSCL-10 score mean among older Hmong participants of 2.23 which is substantially more than the score of 1.85 that indicates clinical depression [30]. Vonnahme et al. (2014) found that $21 \%$ of Bhutanese refugee participants reported a score of 1.75 or greater out of 4 on the depression and anxiety related questions of the HSCL-25 measure, which indicated a positive finding for significant depressive symptoms [31]. Given that additional mental health issues are outside the scope of our study, we recommend future research targeting mental health among long-resettled though less integrated refugee communities.

Many participants reported low levels of physical activity, and furthermore described a diet that includes daily MSG intake. Both these lifestyle behaviors are implicated in chronic disease including hypertension. Current research shows a strong association between high MSG intake and increased BP levels [32, 33]. Health practitioners can suggest alternatives to MSG and promote healthy physical activity to help address the high BP occurrence among Montagnard adults. The survey findings also indicate that the Montagnard participants are low consumers of alcohol and tobacco, and have a daily diet that includes fresh vegetables, rice and only occasional consumption of beverages such as soda. These represent protective behaviors that can play a role in reducing vulnerability to illness and disease. They also report high levels of religious affiliation that can promote social connection and protect health [34]. These community features could be leveraged as focus areas within health outreach and highlighted as strengths on which to build culturally responsive interventions that begin to target the needs of this underserved Asian immigrant/refugee subgroup.

This study has limitations. The convenience sample, recruited through word of mouth and community outreach, may not be representative of this ethno-linguistically diverse Montagnard population. Our church- and community organization-focused outreach may not have included those who are not affiliated with these organizations. Also, members of the smaller, less visible Montagnard tribes (e.g. Koho, Bahnar) may not have attended the churches where we conducted outreach. However, the vast majority of the Montagnard community (individuals affiliated with Rhade, Jarai and Bunong tribal groups) are church members and attend regularly. Further, the number of participants constituting sample size was appropriate for a CBPR study, and represents $1.4 \%$ of the community estimated to be living in NC [35, 36]. Next, despite assiduous efforts to draft culturally sensitive study questions with community informant input, some study participants found the lengthy and detailed questionnaire somewhat onerous, resulting in a lower number of participants for the second part of the questionnaire. The cross-sectional study design can only indicate descriptive patterns. Finally, the health information ascertained in our study is only a partial snapshot of the range of health conditions that the Montagnard community might face. Moreover, our study collected self-reported information on health conditions and supplemented with measures of BP, height and weight, but it was beyond our scope to get professional diagnoses.

\section{New Contributions to the Literature and Community Capacity Building}

Despite shortcomings, the study is, as far as we know, the first to use CBPR to document specific health and lifestyle determinants of chronic disease for this lower SES, limited English proficiency, refugee-origin, now primarily immigrant Asian-American community in the United States. The Montagnards, despite being translocated to the US over 30 years ago, are still poorly integrated into the socioeconomic fabric of this country. Though they mostly reside in largely urban counties in $\mathrm{NC}$ and are within reach of nearby higher education and health care institutions, their education and health profiles remain below national averages. In fact, they remain unknown to many of their longstanding neighbor communities and to regional service providers.

The Montagnards' health profiles are characteristic of medically and socially underserved minority populations, indicating areas of substantial, even critical need. Our use of a CBPR approach to the study allowed us to engage community youth in the contextual interpretation of the findings and dissemination back to the community for their review and use. This occurred via (1) debriefing sessions with community youth, (2) visually appealing and language interpreted slideshows delivered by Montagnard students and faculty researchers during Sunday worship services, (3) short oral presentations by youth at elders' monthly meetings, and (4) community-facilitated discussions with faculty researchers. CBPR also helped to illuminate commonly under-noticed community strengths and points of pride. These include having culturally knowledgeable and community engaged next-generation youth, low predisposition for substance use, strong family networks, a vegetable-rich dietary intake, social connectedness through widespread church affiliation, 
and annual cultural celebrations that include traditional food, music and dance performances by youth and adults alike. These are important ingredients and focal areas for instituting culturally-customized disease prevention interventions and responsive health promotion practice to improve health status and associated outcomes. Chronic disease interventions that incorporate faith-based principles, intergenerational programming and next-generation mentoring would fit with the social and cultural profile of this close knit, tribal community.

In the subsequent years following this study, Montagnard community stakeholders have collaborated with local pastors, congregational nurses, academic faculty (including the co-authors on this paper) and students at area university/college campuses to implement outreach illustrative of culturally tailored family-centered and community-based interventions with this population. For example, there has been congregational programming that offers weekly onsite chronic disease screening, case management and referrals to specialized clinical care by congregational nurses affiliated with the local health system. There have been annual multi-generational health fairs and targeted health outreach activities that have included health insurance enrollment for eligible family members, flu shots, college enrollment information for high school students and young adults; stress reduction activities (e.g. interactive yoga demonstrations, backyard garden tours, and a women's photovoice exhibit) for psychosocial wellbeing; physical activities for children; cultural performances by elders and youth to celebrate tribal and generational heritage; cooking demonstrations with lower sodium ingredients for Montagnard meals; and food distribution and seeds exchanges to support existing fruit and vegetable gardening among families.

At the time of this writing, faculty mentors, public health students and Montagnard leaders have partnered with other minority serving agencies to secure funding for and are distributing food supplies and personal protection equipment (PPE) to Montagnard families impacted by the COVID-19 pandemic [37]. They have also mobilized using social media and through virtual gatherings for tribal language education and awareness outreach about COVID-19 prevention, and have partnered with local community health agencies for targeted immunization outreach to at-risk tribal elders. We (faculty co-authors) continue to recruit and mentor Montagnard youth as next-generation professionals in fields such as public health, nursing, nutrition, social work, human development and family studies, and peace and conflict studies.

Finally, this and other ongoing, longer-term CBPR projects are sustained through involvement with a communitydriven network, the Montagnard/Southeast Asian Community Disparities Research Network [38]. This network functions as a vehicle to increase awareness about Montagnard problems and cultural assets among city officials and service providers. The goal is to build community capacity for applied research and continue to attract interdisciplinary scholars for partnerships and collaborative projects that promote equity, reduce health and social disparities and champion social justice and cultural preservation for this marginalized population.

Acknowledgements We greatly appreciate the willingness to collaborate, time investment and participation of dedicated and hardworking members and advocates of the Montagnard Community. These include the Montagnard Health Professionals, Community Health Workers, Interpreters, the Women's Learning Group, the Montagnard Dega Association, the college students and youth of the Montagnard American Organization, local community advocates, and the congregational nurse, pastors, elders and congregants of the United Montagnard Christian Church, the International Montagnard Bible Church and the Montagnard Central Highlands Church. Thank you all.

Funding Funding for this study was through multiple sources: the Moses Cone Foundation, the Department of Human Development and Family Studies, the School of Health and Human Sciences Office of Research, the Coalition for Diverse Language Communities (CDLC) in the School of Education, the Office of Leadership and Service Learning (OLSL), and the Undergraduate Research, Scholarship and Creativity Office (URSCO), all located within the University of North Carolina Greensboro. Additional funding was through a SENCER (Science Education for New Civic Engagement and Responsibility) Award from the National Center for Science and Civic Engagements.

\section{Declarations}

Conflict of interest The authors declare they have no conflicts of interest.

\section{References}

1. Hickey GC. Free in the forest: ethnohistory of the Vietnamese Central Highlands 1954-1976. New Haven: Yale University Press; 1982.

2. Andresen GD. From Greenwood to Greensboro: Vietnam's Central Highlands and their migration to North Carolina. Master's Thesis, San Diego State University, San Diego, 1988.

3. Siu LM. Developing the first preliminary dictionary of North American Jarai. Master's Thesis, Texas Tech University, Lubbock, 2009.

4. Bailey R. Montagnards: their history and culture. Culture profile. Cultural Orientation Resource Center; 2002. http://www.cultu ralorientation.net/montagnards. Accessed 25 Aug 2020.

5. Kinefugi E. Finding home in migration: Montagnard refugees and post-migration identity. J Int Intercult Commun. 2010;3(3):228-48.

6. Dharod JM, Xin H, Morrison SD, Young A, Nsonwu M. Lifestyle and food-related challenges refugee groups face upon resettlement: do we have to move beyond job and language training programs? J Hunger Environ Nutr. 2013;8:187-99.

7. Corby MR. Developing health communication with displaced populations: an exploration of cultural barriers to health care experienced by Southeast Asian refugees. Elon J Undergrad Res Commun. 2010;1(2):44-50.

8. Xin H, Morrison S, Dharod J, Young A, Nsonwu M. Crosscultural, "Allies" in immigrant community practice: roles of 
foreign-trained former Montagnard health professionals. Health Cult Soc. 2014;6:62-72.

9. Kochhar R, Cilluffo A. Income inequality in the US is rising most rapidly among Asians. Pew Research Center Report; 2018. https:// www.pewsocialtrends.org/2018/07/12/income-inequality-in-theu-s-is-rising-most-rapidly-among-asians/. Accessed 23 Aug 2020.

10. Lueck K. Socioeconomic success of Asian immigrants in the United States. J Ethn Migr Stud. 2018;44:425-38.

11. Minkler M, Wallerstein N. Part one: introduction to communitybased participatory research. In: Community-based participatory research for health. San Francisco: Jossey-Bass; 2003. pp. 5-24.

12. LaVeaux D, Christopher S. Contextualizing CBPR: key principles of CBPR meet the Indigenous research context. Pimatisiwin. 2009;7(1):1

13. Tobias JK, Richmond CA, Luginaah I. Community-based participatory research (CBPR) with indigenous communities: producing respectful and reciprocal research. J Empir Res Hum Res Ethics. 2013;8(2):129-40.

14. Ferreira MP, Gendron F. Community-based participatory research with traditional and indigenous communities of the Americas: historical context and future directions. Int J Crit Pedagogy. 2011;3(3):153-68.

15. Morrison SD, Sudha $\mathrm{S}$, et al. When community calls, we collaborate! Community-based participatory research with the multilanguage Montagnard refugee community. Prog Community Health Partnersh Res Educ Action. 2018;12:179-86.

16. American Heart Association. Clinical practice guidelines. American Heart Association; 2017.

17. Kochanek KD, Murphy SL, Xu J, Arias E. Mortality in the United States, 2013 NCHS Data Brief, No. 178. Atlanta: Centers for Disease Control; 2014.

18. Litwin $\mathrm{H}$. The association between social network relationships and depressive symptoms among older Americans: what matters most? Int Psychogeriatr. 2011;23:930.

19. Jih J, et al. Using appropriate body mass index cut points for overweight and obesity among Asian Americans. Prev Med. 2014;65:1-6. https://doi.org/10.1016/j.ypmed.2014.04.010.

20. Misra A, Dhurandhar NV. Current formula for calculating body mass index is applicable to Asian populations. Nutr Diabetes. 2019;9:3. https://doi.org/10.1038/s41387-018-0070-9.

21. Center for Health Promotion Research. Rapid assessment of physical activity (RAPA). Seattle: University of Washington; 2006. https://depts.washington.edu/hprc/resources/products-tools/rapa/. Accessed 19 Aug 2020.

22. Jonnalagadda SS, Diwan S. Health behaviors, chronic disease prevalence and self-rated health of older Asian Indian immigrants in the US. J Immigr Health. 2005;7:75-83.

23. National Center for Education Statistics. Annual report status and trends in the education of racial and ethnic minorities. National Center for Education Statistics; 2019. https://nces.ed.gov/progr ams/raceindicators/indicator_rfa.asp. Accessed 5 Feb 2021.

24. Southeast Asia Resource Action Center (SEARAC). Data Disaggregation Updated Factsheet. SEARAC; 2019. https://live-searac.
pantheonsite.io/wp-content/uploads/2019/03/2019.02-DataD isagg_UpdatedFactsheet_general_final.pdf. Accessed 5 Feb 2021.

25. Semega, JL, Kollar, MA, Shrider KE, Creamer J. Income and poverty in the United States: 2019. Current Population Reports, P60-270. US Census Bureau; 2020. https://www.census.gov/libra ry/publications/2020/demo/p60-270.html\#: :text=The\%202019\% 20poverty $\% 20$ rate $\% 20$ of, and $\% 20$ Table $\% 20 \mathrm{~B} \% 2 \mathrm{D} 1$. Accessed 6 Feb 2021.

26. City of Greensboro. Analysis of impediments to fair housing choice. Greensboro: City of Greensboro; 2019.

27. Bertrand BM, Dallas MB, Ellington JC. Assessing the health status of an Asian American Group in North Carolina. J Fam Consum Sci. 2012;104(3).

28. Gordon NP, Lin TY, Rau J, et al. Aggregation of Asian-American subgroups masks meaningful differences in health and health risks among Asian ethnicities: an electronic health record based cohort study. BMC Public Health. 2019;19:1551. https://doi.org/10.1186/ s12889-019-7683-3.

29. Office of Minority Health. Mental and behavioral health-Asian Americans. Office of Minority Health; 2019. https://minorityhe alth.hhs.gov/omh/browse. aspx ?lvl=4\&lvlid=54. Accessed $17 \mathrm{Feb}$ 2021.

30. Yang MS, Mutchler JE. The high prevalence of depressive symptoms and its correlates with older Hmong refugees in the United States. J Aging Health. 2020;32(7-8):660-9.

31. Vonnahme LA, Lankau EW, Ao T, Shetty S, Cardozo BL. Factors associated with symptoms of depression among Bhutanese refugees in the United States. J Immigr Minor Health. 2015;17(6):1705-14.

32. Shi Z, et al. Monosodium glutamate is related to a higher increase in blood pressure over 5 years: findings from the Jiangsu Nutrition Study of Chinese Adults. J Hypertens. 2011;29:846-53.

33. Morales C, Rsmevichientong P. Dietary salt-related knowledge, attitude, behaviors and hypertension in a rural Northern Thailand population. Curr Dev Nutr. 2020;4:870-870.

34. Strawbridge WJ, Shema SJ, Cohen RD, Kaplan GA. Religious attendance increases survival by improving and maintaining good health behaviors, mental health, and social relationships. Ann Behav Med. 2001;23:68-74.

35. Connelly LM. Pilot studies. Medsurg Nurs. 2008;17:411-2.

36. Hertzog MA. Considerations in determining sample size for pilot studies. Res Nurs Health. 2008;31:180-91.

37. Al Amin S, Morrison S, Sudha S. Small and invisible, yet strong and impactful-North Carolina's Montagnard Community responds to COVID-19. N C Med J. 2021;981(6):408.

38. The Montagnard/Southeast Asian Community Disparities Research Network. https://www.montagnardda.org/research. Accessed 16 Feb 2021.

Publisher's Note Springer Nature remains neutral with regard to jurisdictional claims in published maps and institutional affiliations. 